\title{
The Physical Aura of Person during Life and after Death in Physics with Real Magnetic Charges
}

\author{
Robert A. Sizov
}

\section{ABSTRACT}

The discovery and study of real magnetic charges, as well as true antielectrons in the structures of substance and their inclusion in basic physical concepts, allowed the author to establish that two physical images correspond to a person: a real human body, i.e., his mass composition (atoms, nucleons, etc.) and the spinor image in the form of its biofield, displayed in the Energo-medium (Energo-ether) that and is the physical Aura. The spinor image or Aura of person is not a simple "photograph" but represents a system of real physical states or fractals in the Energo-medium, which, at the cellular level, are connected with the human body and interact with it at all stages of its existence. It is the physical Aura that is responsible for all the power reactions manifested by the body, providing, for example, "force service" of the activity of the central and autonomic nervous system. The article shows the conditions for the transformation of the Aura into a dead state after the death of a person, and also notes some fractals in its composition that are able to maintain former vital reactions for some time. The external manifestations of such "long-lived" fractals of the Aura, meeting the conditions of identity with a deceased person, can be perceived by some sensitive people. It is the last circumstance that can explain the numerous observations of ghosts and images of deceased people, emerging both in a dream and in reality. At the same time, the ability to form the marked fractals is not an exclusive property of the Aura of dying or deceased people. Such fractals capable of emitting and the quite healthy people in some of life situations. These latter fractals in the surrounding space can be perceived by some people and showing in ordinary dreams.

Keywords: Real magnetic charges, True anti-electrons, Spinor fields, Vortex electromagnetic (gravitational) field, Spinor image of a person, Physical Aura, Biofield of a person, Energo-medium, "Dark energy".

\section{THE PHYSICAL AURA OF A PERSON DURING LIFE AND AFTER DEATH}

The concept of the human Aura in a number of different of esoteric beliefs and oriental religions is explained by the manifestation of the soul and spirit of man. There are numerous cases of using this concept in the framework of so, called pseudoscientific directions, for example, in of representations biofields, dowsing or alternative medicine (see, "Human Aura" Wikipedia).

In official natural science it is believed that the human aura is not a real physical entity and therefore is not a subject of study of any scientific disciplines. However, not everything is so simple, and the above scientific conclusion is not the final verdict.

The discovery and study by the author of real magnetic charges, as well as true anti-electrons in the composition of atoms and substance (not recognized in existing physics), allowed him to establish that the gravitational field is of the vortex electromagnetic field, which is written in the form
Published Online: October 16, 2021

ISSN: 2684-4451

DOI : 10.24018/ ejphysics.2021.3.5.108

Robert A. Sizov *

(e-mail: sizov.robert@ gmail.com)

*Corresponding Author
$\operatorname{rot}[\mathbf{E}-\mathbf{H}]$ and formed by the electromagnetic shells of atoms and nucleons [1]-[5]. It is the gravitational fields emitted by the atoms of the human body that form the basis of its biofield. In addition to the gravitational component, the human biofield includes electric, magnetic, and electromagnetic (the not gravitational) fields that accompany numerous physicochemical processes in the organism.

The author's research has shown that masses (bodies) do not exist empty outer space in but in an Energo-medium (EM) that fills the entire space of the real world and is, for example, the repletion and carrier of a physical essence called the human Aura. The Energo-medium (Energo-phase, Energo-ether) is a super - dense physical medium consisting of its own ultra - small (massless and spinless) particlesenergions moving in different directions at speeds close to light. It is the energion flows are the real physical filling of spinor fields, that is, the fields of charged particles [6], [7]. It is also important to note that the noted particles - energions have the impulse which manifests itself in the processes of force effects of on spinor particles and, consequently, on masses. 
Note 1. The concepts of spinor particles and spinor fields used in the article refer to fundamental particles with such a physical property as spin (literally - "rotation"). It is the spin or the so-called spinority is responsible for such a state of particles as their charged or charge. The physical essence of the spin of particles, in the representations of physics with real magnetic charges, as well as the electric or magnetic properties of fields charges, determined by it, are described in the author's publication (see [6]).

The Energo-medium is of the driving force or global force factor that carries out all primary acts of force in the real World. The primary source of the forces acting on particles and masses are the formations of "Dark Energy" ("DE"), which in the form of oblasts of positive or negative pressure are realized in $\mathbf{E}-\mathbf{M}$ under the impact of spinor fields. It is the "DE" forces that are responsible, for example, both for the dynamics of spinor (charged) particles in atoms and matter, and for the movement of galaxies.

The close concept to the above-described representation of the Energo-medium is the Physical vacuum, i.e., the lowest (ground) energy state of the quantized field, which has zero momentum, angular momentum, and other quantum numbers. The space completely devoid of matter, filled with a field in the described state, is also called a physical vacuum. However, the representation of the Physical Vacuum is fundamentally at odds with the author's concept of the Energo-medium as regards the nature of the physical states or particles that fill the space, as well as the corresponding these zero momentum and angular momentum.

The spinor fields emitted by the masses in the composition of the human body and its constituent biofield, forming the corresponding "prints" or a secondary physical image of a person in the Energo-medium, in much the same way how the human body forms an imprint in the mass of beach sand. However, the image of a person in the E-M, unlike a beach print, is a system of physical states or fractals that correspond to the original at cellular and even at the molecular level. These fractals are closely related to the human body and accompany it at all stages of existence. Thus, two physical entities correspond to a person in the real world: a real human body, including atoms, nucleons, charged particles and a spinor image of a person, in the form of his biofield displayed in the Energo-medium and constituting the physical Aura.

The above-mentioned conclusion about the unified nature of the primary acts of force in the real World carried out by "DE" suggests that all the power processes that are realized in the human body, as well as its external actions, are ultimately realized by the forces of "DE", i.e., through its Aura. Thus, it is the human Aura that carries out the entire power program associated with human vital activity, both at the level of internal physiological processes in the body, and all kinds of external reaction (muscle contractions).

The most complex and functionally saturated part of the human Aura is its area that performs force maintenance of the activity of the human nervous system. It is this area that is responsible for the force implementation of both vegetative processes in the body and its external reactions induced by the central nervous system. In addition, "video" of the most acute life collisions experienced by a person are reflected, encoded, and maintained in Aura. Thus, Aura of the human brain can be defined as a kind of browser, which, in accordance with the physical-biological program embedded in the body, implements both behavioral and autonomous (vegetative) reactions.

For example, muscle reactions (contractions) are realized under the influence of commands of the central or autonomic nervous system. These commands activate the "power browser" and the latter, through the "Dark Energy" forces noted above, forces the corresponding muscles to contract. The mechanics of muscle contractions described above is not difficult to understand, given that the fragments of the Aura responsible for force reactions in the body permeate all its cells, including muscle cells. Given the physics of the formation of the human Aura described above, the behavioral commands of the nervous system are most likely the corresponding complexes of spinor fields.

At the same time, as part of the Aura of a person, there may be fractals that are weakly connected with its gravitational basis, which, depending on physical circumstances, can, "by inertia", function for some time after his death. For the reasons noted above, the destruction of a person's living Aura and its complete transition to a dead mode can take some time, measured in days, and maybe, in some exotic cases, by months or even years All this time, the marked "long-lived" fractals in E-C can show physical activity and influence the surrounding organisms regardless of the body of the deceased person.

The last position raises a very significant question about the interconnectedness human images described above and the possibility of preservation by individual fractals in the composition of the Aura of lifetime physical activity after a person's death.

The main and closely related to the human body in a single geometric space are the fractals of the Aura which are formed by the gravitational fields of atoms. At the same time, due to the different physics underlying the formation of the described primary and secondary images, their unity in space and time is not physically obligatory.

It should be noted that in nature there are cases of separate existence of the marked primary and secondary images. For example, the physical stratification of images is realized in photonic physics, in which a photon is an imprint (secondary image) in the Energy medium of a dynamic act of electric and magnetic charges in a substance, called the birth of a photon. The marked act of charged particles, which can be called the primary image, is itself short-lived. As for its imprint in the Energo-medium, that is, a photon, it is known to be a very long-lived fragment. Thus, in the example with a photon, a physical situation is realized, which can be defined as the separation of the primary and secondary images, or even "life after death".

Of course, a situation in which the Aura of a person after death continues to function in the mode of a full life cycle is absolutely impossible. However, there is no prohibition on the existence and manifestation of independent activity of some fractals that are weakly connected with the basis of the Aura. Such fractals can be, for example, the display in E-M of spinor fields emitted by the human brain at moments of acute life situations, which, first of all, include the moment of his death. Such fractals can persist in the Energo-medium even after the death of a person, and even, likewise a photon, move in space. The noted long-lived fractals, meeting the 
conditions of identity with a deceased person, can be perceived by some sensitive people. It is the last circumstance that can explain the numerous observations of ghosts, the appearance of images of the dead in a dream, and others.

It should be added here that the ability to form and emit the noted so-called long-lived fractals is not an exclusive property of the Aura of dying or deceased people. Such fractals can also be emitted by quite healthy people in some action-packed life situations. These latter manifestations, filling the surrounding space, are selectively perceived by the sensitive people and manifest themselves in ordinary dreams. It should be also understood that all these fractals that are formed in the structures of the human aura are not fiction or illusion, as is commonly believed in the natural sciences, but are based on a completely real physical basis.

In addition to all of the above, a physical effect is known that can be directly related to the existence of a person's physical Aura. This refers to the change in the frequency of optical photons (their reddening) when they pass through the area of the human aura, in our case through the layer of the secondary image of a person in $\mathbf{E - M}$, protruding beyond the geometric limits of the real human body (see Wikipedia "Human Aura").

It is also important to note that the complex of physical imprints in the Energo-medium that determines the described physical Aura is not exclusively the prerogative of a person. Everything that is capable of emitting a spinor field is displayed in the E-M. Of course, the spinor imprint, which forms the gravitational field of an ordinary stone in $\mathbf{E - M}$, cannot be attributed to the image of the Aura. It is appropriate to associate this concept exclusively with the spinor images of living organisms. At the same time, given the high status of man in the natural hierarchy, as well as historical religious traditions, this term can be assigned exclusively to homo sapiens. As for the rest of living beings, their imprints in ES can be called, for example, by such a term as "spinor image" (precisely, beautifully, and scientifically).

Note 2. It is important to note that the status of the photon as a secondary image in the Energy Medium excludes any possibility of its so-called materialization, which, according to reports, is now an obsession. In addition, the very concept of "matter" used in modern physics needs serious correction. The fact is that the concept of matter or the material phase refers exclusively to fundamental electric and magnetic particles with a negative charge. The fundamental representative of matter is the electron. In this case, positively charged particles, both electric and magnetic, constitute the antimaterial phase. Therefore, atoms, nucleons, substance are not matter at all. All these last structural states are masses which are formed by material and antimaterial particles. The fundamental physical property of mass is its ability to induce a gravitational field (see [5]-[7]).

\section{CONCLUSION}

The tragic situation that has developed in physical science as a result of vicious ignoring, for almost 150 years, the true sources of the magnetic field, magnetic charges, as well as true anti-electrons, turned physics, and with it the natural sciences into the "Kingdom of crooked mirrors" [8], [9]. As a result of the noted disregard, atomic shells which in reality are electromagnetic, have been declared purely electronic in physical theory. Physics, which is built on the vicious (electronic) basis described above, can be called electronic or even electrical physics.

Of course, the ignored magnetic and electric particles were forced to replace by such theoretical surrogates as the magnetic moments of electrons and Dirac holes. However, this theoretical "trick" did not solve the problematic situation in physics, but only incredibly complicated and confused its theory.

Among the most famous theoretical "discoveries" generated by vicious electrical physics, one can name: the global expansion of the Universe, a very big bang and a large gap, annihilation of a particle-antiparticle pair, the field and Higgs boson, the representation of the gravitational field in the form of a curved four-dimensional space-time and many others.

It is the vicious electronic physics that is responsible for the negative attitude that has developed in the natural sciences to such a physical entity as the human aura and the reduction of its notions to pseudoscience. The fact is that "thanks" to this vicious physics, all reasonable approaches to such an important component of the real physical World as the Energo-medium were closed whose own particles are the physical filling of the spinor fields. Meanwhile, it is the spinor fields that make up the human biofield and are displayed in E-M what represent such a physical entity as the human Aura. Here we note once again that the basis for classifying the concept of Aura as a pseudoscience is the existing, so-called, electronic physics, which, as the author shows, itself needs serious correction.

\section{APPLICATION}

\section{A. The Main Reasons for Ignoring Real Magnetic Charges in Existing Physics}

Real magnetic charges, as well as true antielectrons, which make up 3/4 of atomic shells, were "buried alive" in existing physics as a result of four basic physical and general human circumstances (see [5]-[7]).

1) The physics of rigid retention of magnetic charges in a substance, which is fundamentally different from the retention of electrons

It is the physics of rigid confinement, magnetic charges and true antielectrons in matter, which is fundamentally different from the retention of electrons, that is the main reason why these particles are very difficult to experimentally isolate from the composition of matter and thoroughly investigate. However, the difficulties of experimental work with magnetic charges in matter do not mean that these particles should have been ignored. It should also be noted that the retention forces of the noted problem particles in the substance increase with an increase in its internal energy. The latter circumstance explains the reasons for unsuccessful attempts to detect these particles in high-energy experiments [10].

2) The primitive-superficial perception of J. Maxwell in 1873 of the results of well-known Oersted experiment

It is precisely, J. Maxwell based on his primitive and superficial impression of the famous Oersted experiment, introduced into physics the vicious myth that the direct sources of the magnetic field are moving electrons. The so- 
called first Maxwell equation $\mathrm{k} \mathbf{J}_{\mathrm{e}}=\operatorname{rot} \mathbf{H}$, in fact, is erroneous, since it involves two different physical processes:

$$
\mathrm{k}_{1} \mathbf{J e}=\operatorname{rot} \mathbf{J}_{\mathrm{g}}
$$

and

$$
\mathrm{k}_{2} \operatorname{rot} \mathbf{J}_{\mathrm{g}}=\operatorname{rot} \mathbf{H}
$$

where $\operatorname{rot} \mathbf{J}_{\mathrm{g}}$ is the vortex orbital current of magnetic charges (a rotating magnetic dipole). The fact is that electrons in the composition of an electric current are exclusively mediators, which are only capable of spinning magnetic dipoles that actually exist in matter (see the first equation). As for the magnetic field, its direct sources are real magnetic charges which, in the compositions of rotating magnetic dipoles, form the well-known vortex magnetic field $\operatorname{rotH}$ (see the second equation) [11].

\section{3) The inertia of erroneous human thinking}

During almost 150 years of the dominance of Maxwell's vicious electric magnetism, theoretical "Himalayas" were built in physical science, on which, starting from "kindergarten", many generations of scientists were brought up. The antagonism to real magnetic charges and true antielectrons in the physical community is so strong that on all the so-called serious information levels, as they say, from the threshold, any movements of thought and even experimental results, associated with the marked problematic particles.

4) The discriminatory policy of the leading physical publications

For example, the articles of author with the results of the study of real magnetic charges, sent to the so-called prestigious journals (Phys.Rev, Nature, etc.) were immediately repudiate, with expert opinions which, in general, can be described by the expression: this can never be. It is the leading physics publications they stand guard over the existing theoretical physics, creating numerous "chippers" that prevent the penetration of views on their pages that are alien to official physical dogmas.

Of course, it is possible to understand the noted "defensive" reaction of the Editorials and their experts, since the introduction into fundamental physics of real magnetic charges, as well as true antielectrons, presupposes a radical revision of the entire theoretical arsenal of physics created in 150 years of ignoring these particles. By the way, this arsenal accounts for at least $70 \%$ of the content of the leading physical journals for all the years of the dominance of vicious electric magnetism in the sciences, i.e., counting from 1873.

At the same time, as it usually happens in life, any delusion, even such a grandiose one, as described above, sooner or later passes. Therefore, in the future we still have a very "fascinating" spectacle with the "fall" of modern physical theory from the conquered "peaks". Of course, only those who can live up to this moment will be able to see this. http://dx.doi.org/10.4236/jmp.2015.68106.

[2] Robert A. Sizov, "The Experiments Detecting of Real Magnetic Charges in Structures of Atoms and Substance," Journal of Modern Physics, vol. 11, pp. 1245-1261, 2020. https://doi.org/10.4236/jmp.2020.118078.

[3] Robert A. Sizov, "Experimental Ferrogravitational Field around Untwisting Closed Superconductor," Journal of Modern Physics, vol. 11, pp. 1807-1826, 2020. DOI: 10.4236/jmp.2020.1111113.

[4] Robert A. Sizov, "The magneto-motive force, currents of magnetic and electric charges in static superconductor and them vortex spinor fields," Journal Current Trend in Physics Research and Applications, vol. 2(1), pp. 1-10, 2021. https://katalystpub.com/jctpra-articles-inpress/.

[5] Robert A. Sizov, "Electromagnetic Shells of Atoms and the Periodic System of Elements," Journal of Modern Physics, vol. 7, pp. 2374 2397, 2016. http://dx.doi.org/10.4236/jmp.2016.716206.

[6] Robert A. Sizov, "World Physical Triad: Matter, Antimatter and "Dark Energy" in the Processes of Climatic Changes on the Earth," Journal of Modern Physics, vol. 7, pp. 558-572, 2016. http://dx.doi.org/ 10.4236/jmp.2016.76059.

[7] Robert A. Sizov, "Real Magnetic Charges in the Substance, Ferrogravitation and Technical Levitation," Journal of Modern Physics, vol. 6, pp. 1591-1601, 2015. http://dx.doi.org/10.4236/jmp.2015.611161.

[8] R. A. Sizov, "The electrical magnetism of Maxwell (1873) is the "crooked mirror" of physical science.,"Applied Physics Research, vol. 11, no. 3, pp. 49-55, 2019. http://dx.doi.org/10.5539/apr.v11n3p49.

[9] Robert A. Sizov, "'Particle of God" as insulting of the Divine Principle," International Journal of Theoretical \& Computational PhysicS, 2021. DOI: doi.org/10.47485/2767-3901.1010.

[10] Robert A. Sizov, "“Electrical" and "Magnetic" Worlds in Universe," Journal of Modern Physics, vol. 8, pp. 1072-1086, 2017. DOI: 10.4236/jmp.2017.87069.

[11] Robert A. Sizov, "Great Mistake of Great J. C. Maxwell," Applied Science and Innovative Research ISSN (Print), Vol. 4, No. 1, 2020. http://dx.doi.org/10.22158/asir.v4n1p32.

Robert A. Sizov is Doctor of Physical and Mathematical Sciences, specialist in the oblast of general physics with real magnetic charges. Currently, he works as an individual researcher. Extended information about his research on the topic: real magnetic charges in atoms and matter can be found by typing in Google: Robert Sizov physics.

\section{REFERENCES}

[1] Robert A. Sizov, "Real magnetic poles (magnetic charges) in the Physics of magnetism, gravitation and levitation," Journal of Modern Physics, vol. 6, pp. 1013-1022, 2015. 